\title{
Vibrational excitation cross sections of methane by electron impact
}

\author{
T W Shyn \\ Space Physics Research Laboratory, The University of Michigan, Ann Arbor, MI 48109, \\ USA
}

Received 9 April 1991, in final form 20 August 1991

\begin{abstract}
The vibrational excitation cross sections of methane (ground state) have been measured by electron impact. A crossed-beam method was used. The incident energies used were $5.0,7.5,10.0$ and $15 \mathrm{eV}$ and the angular range covered was from $24^{\circ}$ to $156^{\circ}$ at $12^{\circ}$ intervals. The present results were compared with previous measurements by Tanaka et al and some considerable discrepancies were found.
\end{abstract}

\section{Introduction}

Methane has been detected in the atmospheres of Jupiter, Saturn, Uranus and Neptune as well as in Titan's atmosphere [1]. A proper understanding of these atmospheres requires a knowledge of electron impact cross sections of methane including the vibrational excitation.

Rohr [2] measured vibrationally elastic scattering cross sections in the energy range below $10 \mathrm{eV}$ and vibrational excitation cross sections from threshold to about $4 \mathrm{eV}$ over the angular range $10^{\circ}-120^{\circ}$. Sohn et al [3] also measured the elastic and inelastic cross sections for collision energies from 0.1 to $1.8 \mathrm{eV}$ in order to study the shapes of the excitation functions of the four vibrational modes. The most extensive measurements of vibrational excitation cross sections were made by Tanaka et al [4]. They measured the differential cross sections of vibrational excitations $\nu_{2,4}\left(\nu_{2}\right.$ and $\nu_{4}$ modes) and $\nu_{1,3}$ ( $\nu_{1}$ and $\nu_{3}$ modes) of $\mathrm{CH}_{4}$ in the energy range 3-20 eV, over the angular range of $30^{\circ}-140^{\circ}$.

The present work presents results of measurements of the differential vibrational excitation cross sections from the ground state of methane in the energy range $5-20 \mathrm{eV}$ over the angular range $24^{\circ}-156^{\circ}$.

\section{Apparatus and procedure}

A schematic diagram of the apparatus for the present mesurements is the same as for the measurement of the elastic cross sections of $\mathrm{CH}_{4}$ published previously [5]. A detailed description can be found elsewhere [5-7]. Briefly, differentially pumped upper and lower chambers contain the apparatus. A neutral $\mathrm{CH}_{4}$ beam source is located in the upper chamber. The vertically collimated $\mathrm{CH}_{4}$ beam from the source enters the lower chamber through a double skimmer located between the two chambers. The 
monoenergetic electron beam source and an electron energy analyser system (in a horizontal plane) are located in the lower chamber.

The electron beam source consists of an electron gun, a $127^{\circ}$ energy selestor, two electron lenses and two electron beam deflectors. It is rotatable from $-90^{\circ}$ to $+160^{\circ}$ continuously. During the present set of measurements, the electron source produces an electron current of $10^{-9} \mathrm{~A}$ with an energy resolution of $80 \mathrm{meV}$ (FWHM) at $2 \mathrm{eV}$ incident energy and better resolution for higher energies. The resolution of measurements by Tanaka et al was claimed to be $30 \mathrm{meV}$ but they had a single analyser in their detector system. Our fixed detector system consists of two electrostatic energy analysers placed in series, two electron lenses and a channeltron electron multiplier. This double analyser system has an energy resolution of better than $60 \mathrm{meV}$ and reduces the background signal compared to our previously used single analyser system by a factor of approximately 100 . The solid angle of detection is about $5 \times 10^{-4} \mathrm{sr}$. The divergence angle of the incident electron beam is $\pm 3^{\circ}$.

The incident electron beam of a given energy intersects with the collimated neutral beam in an interaction region. The scattered electrons from the methane beam at a given angle are detected by the electron channeltron multiplier after energy analysis. Thus, the energy loss spectrum of the scattered electrons is obtained at a given scattering angle at an incident energy. This procedure is repeated for different angles and incident energies. A typical energy loss spectrum at $7.5 \mathrm{eV}$ impact energy is shown in figure 1 . The energy resolution of the present apparatus $(\approx 80 \mathrm{meV})$ is not sufficient to resolve the two sets of two individual modes $\left(\nu_{1}\right.$ and $\nu_{3}, \nu_{2}$ and $\left.\nu_{4}\right)$. The ratio of the cross section for the sum of two modes with respect to the elastic peak is determined for each spectrum. The absolute excitation cross section at a given angle is obtained by multiplying the relative vibrational cross sections and the absolute elastic cross section which was measured by the present author [5]. At a given incident energy, the angular

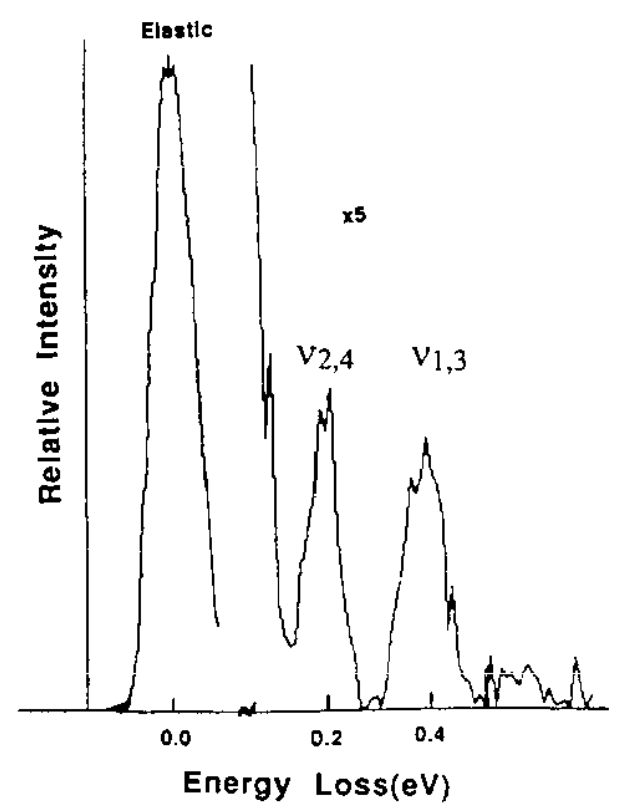

Figure 1. Energy loss spectrum at $5 \mathrm{eV}$ electron impact energy for a scattering angle of $120^{\circ} . \nu_{2,4}$ and $\nu_{1,3}$ are vibrational excitation modes. 
distribution measurement was repeated more than four times over a six month period in order to ensure the reproducibility of the present measurements.

The impact energy scale is' calibrated against the $19.3 \mathrm{eV}$ resonance of He. Three sets of Helmholtz coils reduce stray magnetic fields down to less than $20 \mathrm{mG}$ in all directions near the interaction region.

\section{Experimental results}

We have measured vibrational excitation cross sections from the ground state at incident energies of 5.0, 7.5, 10 and $15 \mathrm{eV}$. A crossed-beam method was used. The present results have been placed on an absolute scale by normalizing to the elastic cross sections of $\mathrm{CH}_{4}$ measured by Shyn and Cravens, except for the impact energy of $7.5 \mathrm{eV}$. Elastic cross sections of Tanaka et al have been used for the normalization process of our elastic cross section for $7.5 \mathrm{eV}$ impact at $96^{\circ}$. The final results are shown in table 1 (a) and (b).

Table 1. Differential vibrational excitation cross section of $\mathrm{CH}_{4}$. (a) $\mathrm{d} \sigma\left(\nu_{2,4}\right) / \mathrm{d} \Omega\left(10^{-18} \mathrm{~cm}^{2} \mathrm{sr}^{-1}\right)$. (b) $\mathrm{d} \sigma\left(\nu_{1,3}\right) / \mathrm{d} \Omega\left(10^{18} \mathrm{~cm}^{2} \mathrm{sr}^{-1}\right)$. Numbers in parentheses are extrapolated data points.

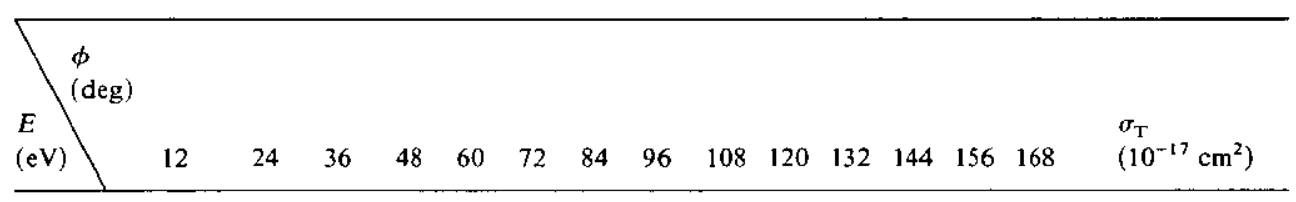

(a)

$\begin{array}{cccccccccccccccc}5.0 & (8.8) & 6.2 & 3.3 & 2.7 & 2.2 & 2.1 & 2.0 & 2.0 & 2.3 & 2.6 & 2.6 & 1.6 & 1.1 & (0.74) & 3.2 \\ 7.5 & (7.8) & 6.7 & 5.8 & 4.1 & 3.5 & 3.1 & 3.5 & 4.1 & 4.4 & 3.9 & 4.4 & 3.8 & 3.6 & (3.5) & 5.2 \\ 10 & (7.0) & 5.1 & 3.1 & 2.4 & 2.0 & 1.9 & 1.6 & 1.7 & 1.6 & 2.8 & 3.1 & 2.7 & 2.3 & (2.0) & 3.1 \\ 15 & (2.9) & 2.7 & 2.5 & 2.3 & 1.7 & 1.4 & 1.4 & 1.4 & 1.5 & 1.6 & 2.0 & 2.0 & 2.0 & (2.1) & 2.3\end{array}$

(b)

$\begin{array}{llllllllllllllll}5.0 & (14.0) & 9.7 & 6.6 & 5.5 & 4.7 & 3.9 & 5.0 & 4.8 & 4.3 & 3.7 & 2.6 & 1.8 & 1.4 & (0.95) & 5.7\end{array}$

$\begin{array}{llllllllllllllll}7.5 & (22.0) & 14.5 & 10.6 & 6.4 & 5.7 & 4.9 & 5.2 & 5.6 & 4.5 & 4.0 & 4.2 & 4.5 & 5.3 & (6.0) & 7.7\end{array}$

$\begin{array}{llllllllllllllll}10 & (11.0) & 6.4 & 4.0 & 3.7 & 3.2 & 2.8 & 2.0 & 1.9 & 2.0 & 2.6 & 2.6 & 2.9 & 3.8 & (4.5) & 3.9\end{array}$

$\begin{array}{llllllllllllllll}15 & (2.1) & 2.0 & 1.8 & 1.7 & 1.4 & 1.4 & 1.3 & 1.1 & 1.1 & 1.5 & 2.1 & 2.1 & 2.4 & (2.7) & 2.0\end{array}$

The statistical uncertainty of the data points is less than $\pm 20 \%$ for the $\nu_{1,3}$ mode and less than $\pm 25 \%$ for the $\nu_{2,4}$ mode. Overall uncertainty of the present results is $\pm 25 \%$ for $\nu_{\mathrm{t}, 3}$ mode, and $\pm 29 \%$ for $\nu_{2,4}$ mode including the uncertainty in the $\mathrm{CH}_{4}$ elastic cross sections $( \pm 14 \%)$.

Figure 2 shows differential excitation cross sections at $5 \mathrm{eV}$ impact along with those of Tanaka et al. Agreement between the present results and those of Tanaka et al for $\nu_{1,3}$ is good. However, the results of $\nu_{2,4}$ cross sections are not in good agreement even though our previous measurement of elastic cross sections [5] and those of Tanaka et al are in good agreement. The present results are larger than those of Tanaka by a factor of two, approximately, except near $120^{\circ}$.

Figure 3 is the same as figure 2 except for $7.5 \mathrm{eV}$ impact energy. There is a relatively good agreement between the present results and those of Tanaka et al except for the forward angle for the $\nu_{1,3}$ mode. The present results have larger forward scattering 


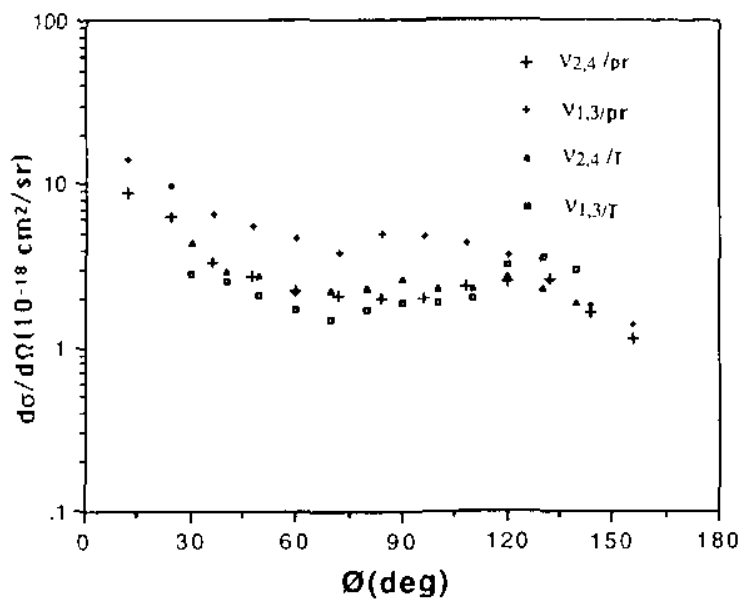

Figure 2. Angular distribution of vibrational excitation cross sections at $5 \mathrm{eV}$ impact energy (designated PR). The cross sections of Tanaka et al are also shown (designated $\mathrm{T}$ ).

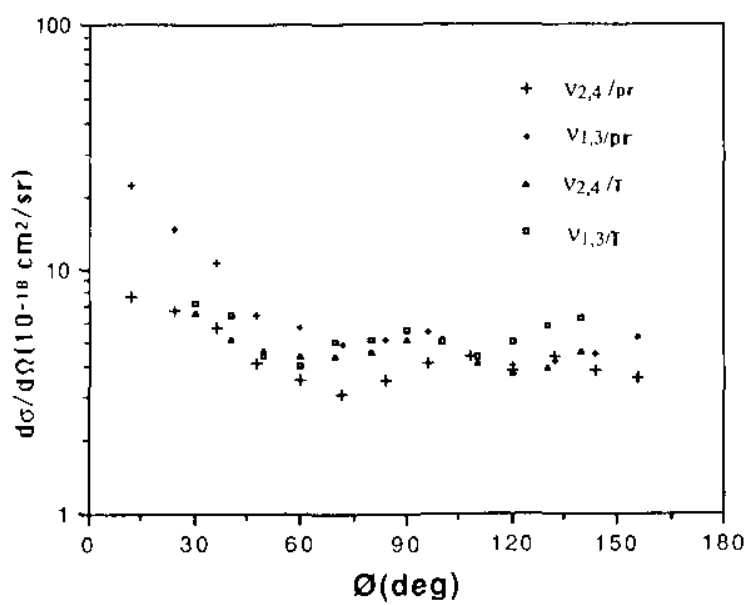

Figure 3. Same as figure 2 except for an incident energy of $7.5 \mathrm{eV}$

than those of Tanaka et al by more than a factor of two. This may be due to larger forward scattering cross section of our previous measurement than those of Tanaka et al.

The results for 10 and $15 \mathrm{eV}$ impact energies agree with those of Tanaka et al within the experimental uncertainty.

Figure 4 shows the integrated cross sections of both modes along with those of Tanaka et al. The integrated cross sections were obtained by integrating the differential cross sections over solid angles after an exponential extrapolation to $0^{\circ}$ and $180^{\circ}$. There is a good agreement between the present results and those of Tanaka et al above $10 \mathrm{eV}$ impact energy but some disagreement exists below $10 \mathrm{eV}$. The present measurements show stronger resonance effects for the $\nu_{1,3}$ mode and less effects for the $\nu_{2,4}$ mode near $7.5 \mathrm{eV}$ than those of Tanaka et al. The present integrated values for the $\nu_{1,3}$ mode are larger than those of Tanaka et al by about a factor of two. 


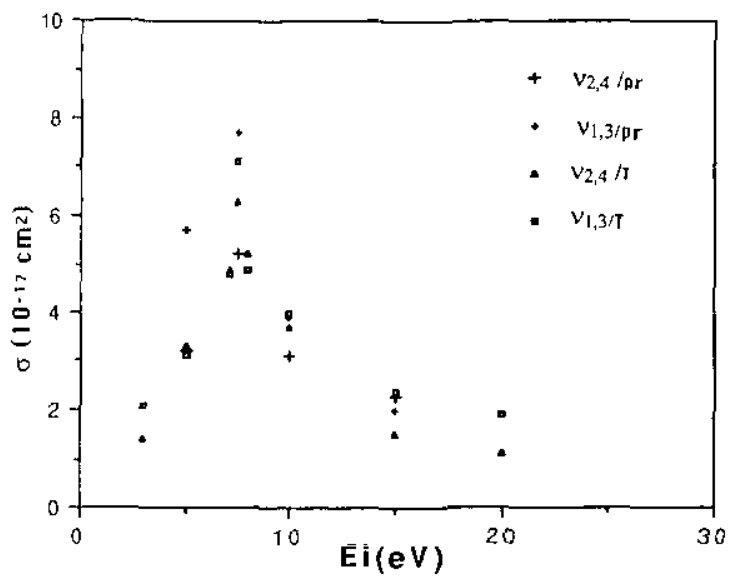

Figure 4. Integrated vibrational excitation cross sections of $\mathrm{CH}_{4}$ (designated PR) along with those of Tanaka $e t$ al (designated $T$ ).

\section{Acknowledgment}

The author is very grateful to Professor Thomas E Cravens for reading the manuscript and supplying many comments. This work was supported by NASA Grant No NAGW938.

\section{References}

[1] Hunter D M, Tomasko M G, Fluser F M, Samuelson R E, Strobel D F and Stevenson D J 1984 Titan Saturn ed J Cehrels and M S Matthews (Tucson, AZ: University of Arizona Press) p 67 !

[2] Rohr K 1980 J. Phys. B: At. Mol. Phys. 132919

[3] Sohn W, Jung K and Ehrhardt H 1983 J. Phys. B: At. Mol. Phys. 16891

[4] Tanaka H, Kubo M, Onodera N and Suzuki A 1983 J. Phys. B: At. Mol. Phys. 162861

[5] Shyn T W and Craven T E 1990 J. Phys. B: At. Mol. Opt. Phys. 23293

[6] Shyn T W, Cho S Y and Craven T E 1988 Phys. Rev. A 38678

[7] Shyn T W and Cho S Y 1989 Phys. Rev. A 401315 